\title{
Le marigot houët à Bobo-Dioulasso : une question de santé publique?
}

\author{
Yacouba OUATTARA ${ }^{1}$, Issaka GUIGUEMDE ${ }^{1,2^{*}}$, Françoise DIENDERE ${ }^{2}$, \\ Nassourou TALL ${ }^{1}$, Soumaïla N'DIAYE ${ }^{1,4}$, Jean DIARRA ${ }^{3}$, Ardjouma SANOU ${ }^{4,5}$ et \\ Abdouraman BARY ${ }^{2}$ \\ ${ }^{I}$ Centre d'Etude pour la Promotion, l'Aménagement et la Protection de l'Environnement (CEPAPE) \\ 03 BP 7021 Université de Ouagadougou, Burkina Faso. \\ ${ }^{2}$ Laboratoire de Chimie Analytique, de Radiochimie et d'Electrochimie (LACARE), UFR/SEA 03 BP 7021 \\ Université de Ouagadougou, Burkina Faso. \\ ${ }^{3}$ Service Qualité- Eau de l'Office National de l'Eau et de l'Assainissement (ONEA) \\ 01 BP 170 Ouagadougou 01, Burkina Faso. \\ ${ }^{4}$ Commune de Bobo Dioulasso;01 BP 383 Bobo-Dioulasso, Burkina Faso. \\ ${ }^{5}$ Direction de l'Action Sanitaire de la Municipalité de Bobo Dioulasso 01 BP 383 Bobo-Dioulasso, \\ Burkina Faso. \\ *Auteur correspondant ; E-mail : issakguig@yahoo.fr; Tél. (00226) 76651388
}

\section{RESUME}

Le marigot Houët, un affluent de la rivière Kou, traverse l'intérieur de la ville de Bobo-Dioulasso du Sud au Nord. Il abrite des poissons silures qui sont considérés comme sacrés par les populations riveraines et sert aussi de source d'activités socio-économiques pour ces populations. L'objectif de notre étude est de faire un état des lieux, partant, des fonctions du marigot, afin de contribuer à la protection de son écosystème et à l'amélioration de la santé et du cadre de vie des populations riveraines. Pour notre étude, nous avons fait des prélèvements d'échantillons d'eau en sept (7) points du marigot couvrant toutes les activités socioéconomiques. Les analyses des paramètres physicochimiques de pollution $(\mathrm{pH}$, conductivité, turbidité, matières en suspension, ortho-phosphates, nitrates et nitrites, sulfates et chlorophylle «a »), de la demande chimique en oxygène $(\mathrm{DCO})$, de la demande biologique en oxygène $\left(\mathrm{DBO}_{5}\right)$, des paramètres microbiologiques $(E$. coli, coliformes fécaux, streptocoques fécaux) et des paramètres organoleptiques montrent que l'eau du Houët est sujette à une pollution domestique et industrielle.

(c) 2012 International Formulae Group. All rights reserved.

Mots clés: écosystème, pollution, silures, eau.

\section{INTRODUCTION}

Le bassin hydrographique du Mouhoun, appartient au bassin international de la Volta. Sa superficie totale est estimée à $412,350 \mathrm{~km}^{2}$, pour $90,810 \mathrm{~km}^{2}$ en territoire burkinabè. Il prend sa source sur les plateaux gréseux de la région des Hauts-Bassins. Le nombre de sources du bassin du Mouhoun a été estimé par le programme Ressources en Eau Souterraine de l'Ouest (Wellens et al., 2007) du Burkina à environ 183 sources dont 142 pérennes. Elles se situent surtout dans la 
partie Sud du bassin. Dans la zone sédimentaire qui compte 92 sources dont 84 pérennes et 8 temporaires, les eaux de ces sources sont drainées par le Haut Mouhoun et ses affluents dont le Kou (AEM, 2012). Le marigot Houët, un affluent du Kou, traverse l'intérieur de la ville de Bobo-Dioulasso du Sud au Nord. Il abrite des silures qui malgré leur très haut degré de résistance, subissent des fois des cas de mortalités massives liées à la pollution des eaux (DGACV/MECV, 2005). Le présent article porte sur le marigot Houët, car il présente de multiples intérêts. En effet, le marigot abrite en plus des silures sacrés de Bobo-Dioulasso, des cultures maraîchères irriguées et fruitières et sert aussi de source d'activités socioéconomiques pour la population riveraine. Les eaux du marigot Houët sont particulièrement dégradées aujourd'hui, en raison des lourdes charges de pollution que ce dernier reçoit toute l'année (Banhoro, 2007 ; Belghyti, 2009 ; Mama, 2010). Ces eaux ont pourtant connu un début d'épuration avec la mise en œuvre du plan stratégique d'assainissement de la ville de Bobo-Dioulasso et particulièrement la mise en service des égouts ainsi que de la Lagune de Dogona en août 2008. Notre étude est menée dans le but de déterminer l'état de pollution du marigot Houët, ce qui nous permettra de connaître l'état des lieux des différentes fonctions du marigot (eau du marigot utilisée comme domestique, comme eau de baignade et comme eau piscicole), afin de contribuer à la protection de son écosystème et à l'amélioration de la santé et du cadre de vie des populations riveraines, en particulier de Bobo-Dioulasso et du sous bassin du Kou en général.

La stratégie de l'étude a reposé sur une approche méthodologique fondée sur les observations directes sur le terrain et des analyses en laboratoire. Dans la partie discussion, nous comparons les valeurs de quelques paramètres avec d'autres résultats antérieurs, pour faire un bilan de l'impact de l'assainissement collectif sur le marigot Houët.

\section{MATERIEL ET METHODES \\ Echantillonnage}

Pour nos analyses, sept (07) points (P1 à P7) ont fait l'objet de prélèvements des échantillons d'eau. Des bouteilles préalablement stérilisées ont été utilisées pour la détermination des paramètres microbiologiques et des flacons en polyéthylène pour celle des paramètres physicochimiques. Les points retenus sont les suivants :

- P1 en amont du pont Louveau, à proximité des producteurs maraîchers et des pépiniéristes et d'un site d'offrande aux silures sacrés ;

- P2 à l'est du Musée Communal, à proximité des producteurs maraîchers et des pépiniéristes, mais aussi de l'ancienne frayère des silures ;

- P3 à l'est du Jardin Zoologique et à proximité des producteurs maraîchers ;

- P4 à Dioulassoba où l'on trouve la Mare aux Silures Sacrés ;

- P5 à Wara-wara ; avec le point (P4), ce site connait un fort développement de l'activité domestique à travers la lessive et le lavage de vaisselle ;

- P6 à l'ouest de la zone maraîchère de Dogona, où les revendeuses de produits maraîchers nettoient leurs marchandises ;

- $\quad$ P7 après l'exutoire de la Lagune. La zone entre ce point et le point P6 constitue un lieu de baignade pour les enfants des riverains.

La logistique terrain et le personnel de soutien ont été assurés par la Direction de l'Action Sanitaire de la Municipalité de BoboDioulasso.

\section{Instrumentation et méthodes}

- Dans cette étude, les seules déterminations in situ sont celles de la température, du pH et de la conductivité ; 
- Le pH a été déterminé par électrochimie avec le pH-mètre HANNA instruments HI 98230 (méthode conforme à la norme française NFT 90-008) ;

- La température et la conductivité ont été mesurées avec le Multi paramètres WTW multi 340i (méthode conforme à la norme française NF T 90-031) ;

- La turbidité a été mesurée avec la méthode basée sur le mesurage de la lumière diffusée avec le Turbidimètre WTW Turb 550 IR (méthode conforme à la norme française NF ISO 7027) ;

- Les Matières en Suspension, les orthophosphates, les nitrates et les nitrites ainsi que les sulfates ont été mesurés par spectrophotométrie avec le Spectrophotomètre Lange Hach DR 3800 (Méthode photométrique) ;

- La chlorophylle 'a' par spectrophotométrie après filtration sur disques en filtres de verre. L'extraction de la chlorophylle 'a' s'est faite dans l'éthanol et la mesure avec le Spectrophotomètre UV-VIS Shimadzu UV1601 ;

- $\mathrm{La} \mathrm{DBO}_{5}$ a été mesurée par la méthode d'incubation pendant cinq jours à l'aide d'un appareil Oxitop ${ }^{\mathrm{R}} \mathrm{WTW}$, la mesure faite par des sondes de pression électroniques résistantes ;

- La DCO a été obtenue par la méthode au dichromate de potassium avec un réacteur de type RECOD/19 Test ;

- Les microorganismes d'impact sanitaire (coliformes fécaux, E. coli, les streptocoques fécaux) ont été déterminés par la méthode de filtration sur membrane; les milieux de culture RapidE.coli, chromocult et bile esculine ont été utilisés pour le dénombrement des indicateurs bactériens respectifs. L'incubation a été faite à $44,5{ }^{\circ} \mathrm{C}$ pendant 24 $48 \mathrm{~h}$ dans une étuve thermostatée au laboratoire ;

- Les paramètres organoleptiques ont été déterminés par observation.

\section{RESULTATS}

Le Tableau 1 indique les résultats des observations organoleptiques effectuées sur les échantillons récoltés. Le Tableau 2 et les Figures 1 à 15 indiquent les résultats des analyses physico-chimiques et des analyses microbiologiques effectuées sur les échantillons.

Tableau 1: Résultats des analyses des paramètres organoleptiques.

\begin{tabular}{lcccc}
\hline $\begin{array}{l}\text { Point de } \\
\text { prélèvement }\end{array}$ & $\begin{array}{c}\text { Couleur de } \\
\text { l'eau }\end{array}$ & Odeur de l'eau & $\begin{array}{c}\text { Importance des } \\
\text { déchets solides } \\
\text { présents }\end{array}$ & Autre caractéristique \\
\hline P1 & Claire & Non perceptible & Important & \\
P2 & Trouble & Non perceptible & Faible & Déchets plastiques \\
P3 & Claire & Non perceptible & Faible & $\begin{array}{c}\text { Dén d'ordure à proximité } \\
\text { P4 }\end{array}$ \\
Trouble & Peu perceptible & Très important & $\begin{array}{c}\text { du lit du marigot } \\
\text { Défécation aux alentours et } \\
\text { dans le lit }\end{array}$ \\
P5 & Trouble & Peu perceptible & Très important & Feuilles de légumes \\
P6 & Peu claire & Non perceptible & Important & \\
P7 & Trouble & Forte & Peu important & \\
\hline
\end{tabular}


Tableau 2: Résultats des analyses des paramètres microbiologiques.

\begin{tabular}{|c|c|c|c|c|c|c|c|c|}
\hline \multirow{2}{*}{$\begin{array}{l}\text { Paramètres } \\
\text { microbiologiques }\end{array}$} & \multirow[t]{2}{*}{ Unités } & \multicolumn{7}{|c|}{ Echantillon } \\
\hline & & P1 & $\mathbf{P 2}$ & P3 & P4 & P5 & P6 & P7 \\
\hline $\begin{array}{l}\text { E. Coli } \\
\text { Coliformes }\end{array}$ & $\mathrm{U}$ & 1600 & 4700 & 1400 & $>10000$ & $>10000$ & 2100 & $>10000$ \\
\hline $\begin{array}{l}\text { fécaux } \\
\text { Streptocoques }\end{array}$ & $\mathrm{U}$ & $>10000$ & 9100 & $>10000$ & 6400 & $>10000$ & $>10000$ & 2600 \\
\hline fécaux & U & 1300 & 6300 & 2000 & $>10000$ & $>10000$ & $>10000$ & $>10000$ \\
\hline
\end{tabular}

Tableau 3: Tableau comparatif des valeurs de la température, du $\mathrm{pH}$, de la conductivité et de la turbidité avec le temps.

\begin{tabular}{lcccccc}
\hline Année & $\mathbf{2 0 0 1}$ & \multicolumn{2}{c}{$\mathbf{2 0 0 7}$} & \multicolumn{2}{c}{$\mathbf{2 0 1 2}$} \\
\hline Echantillon & $\begin{array}{c}\text { Zone en } \\
\text { aval (P7) }\end{array}$ & $\begin{array}{c}\text { Zone en } \\
\text { amont (P1) }\end{array}$ & $\begin{array}{c}\text { Zone en } \\
\text { aval (P7) }\end{array}$ & $\begin{array}{c}\text { Plus petite } \\
\text { valeur }\end{array}$ & $\begin{array}{c}\text { Valeur } \\
\text { supérieure } \\
\text { en ville }\end{array}$ & $\begin{array}{c}\text { Plus grande } \\
\text { valeur }\end{array}$ \\
\hline \multicolumn{7}{c}{ Paramètres } \\
\hline $\mathrm{T}^{\circ}\left({ }^{\circ} \mathrm{C}\right)$ & 27,15 & 30 & 30 & $25,7(\mathrm{P} 2)$ & $27,7(\mathrm{P} 6)$ & $29(\mathrm{P} 7)$ \\
$\mathrm{pH}$ & 6,8 & 7,05 & 7,1 & $6,8(\mathrm{P} 4)$ & $7(\mathrm{P} 2)$ & $7,2(\mathrm{P} 3 \mathrm{et} \mathrm{P} 7)$ \\
$\begin{array}{l}\text { Conductivité } \\
(\mu \mathrm{S} / \mathrm{cm})\end{array}$ & 273 & 305 & 520 & $225(\mathrm{P} 4)$ & $371(\mathrm{P} 6)$ & $1145(\mathrm{P} 7)$ \\
Turbidité (FNU) & & 8,6 & 150 & $14,34(\mathrm{P} 3)$ & $27,26(\mathrm{P} 4)$ & $323(\mathrm{P} 7)$ \\
\hline
\end{tabular}

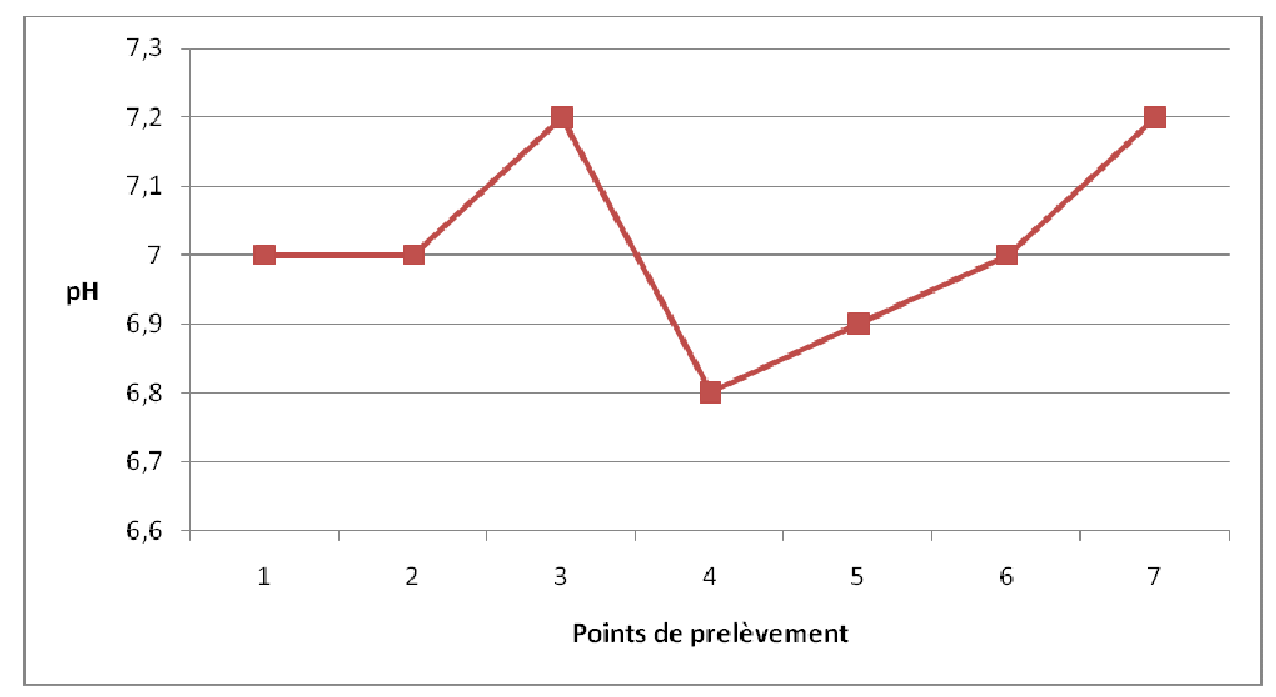

Figure 1: Valeurs du pH en fonction des points de prélèvement. 


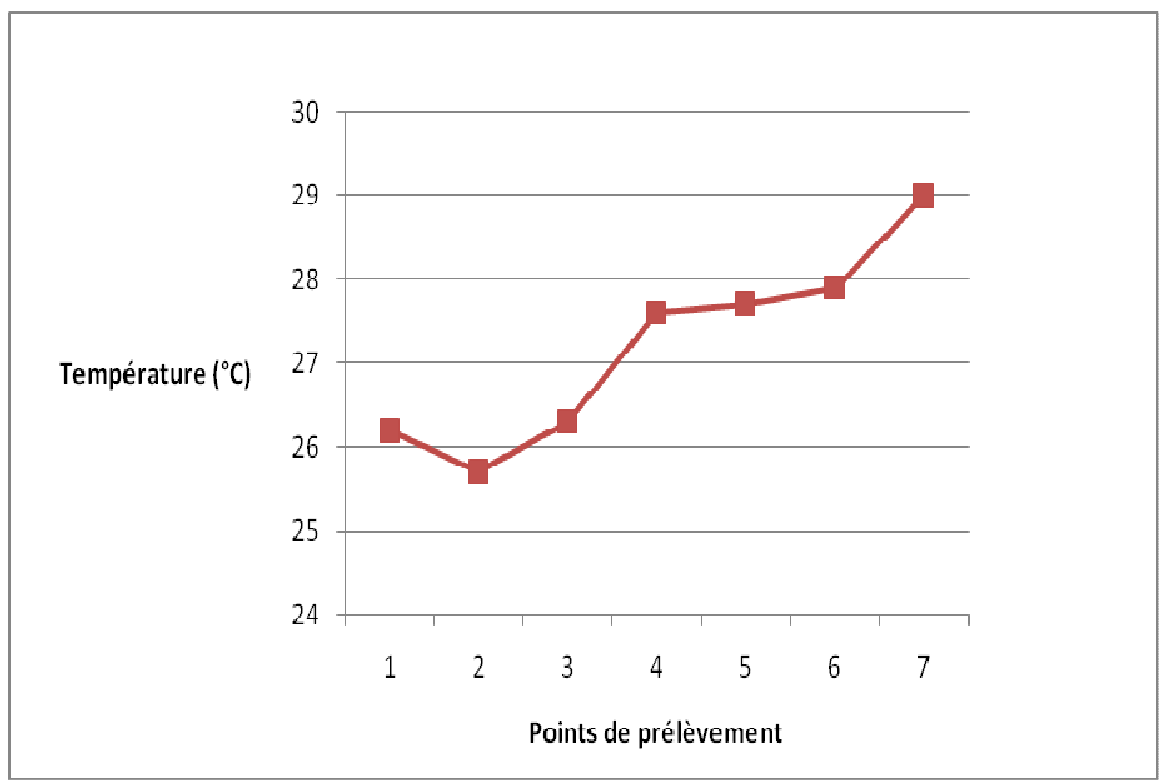

Figure 2: Valeurs de la température en fonction des points de prélèvement.

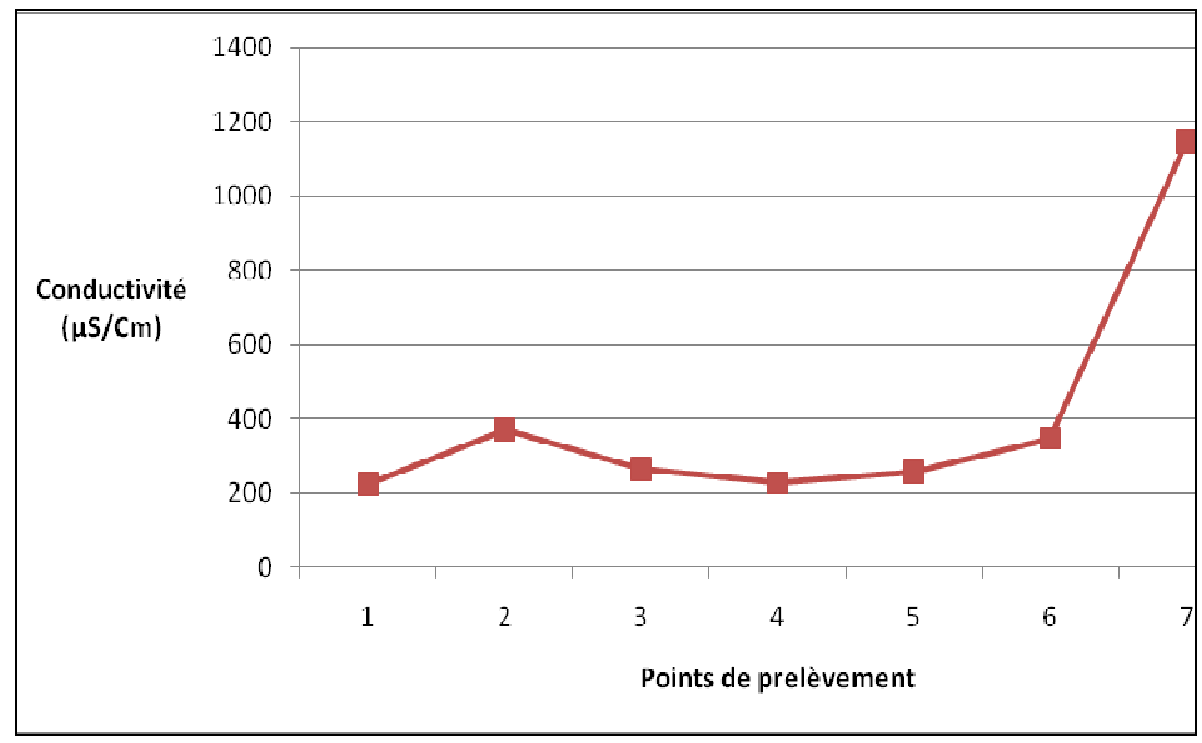

Figure 3: Valeurs de la conductivité en fonction des points de prélèvement. 

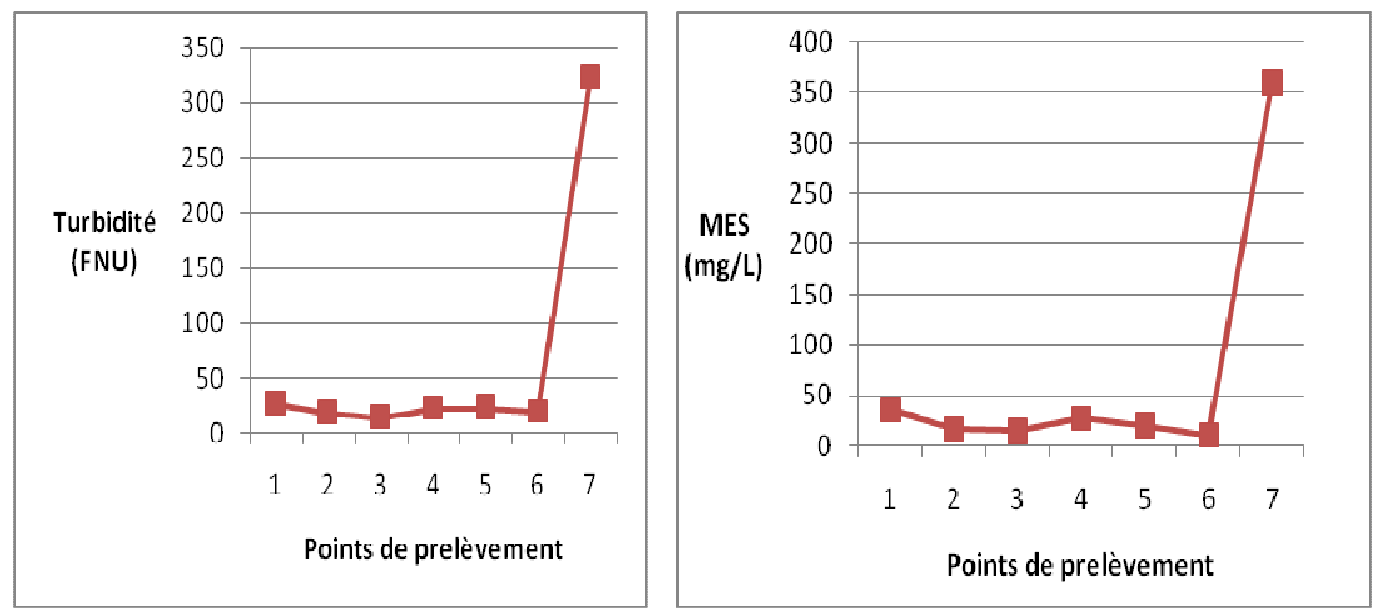

Figure 4: Valeurs de la turbidité en fonction Figure 5: Valeurs des MES en fonction des points des points de prélèvement. de prélèvement.
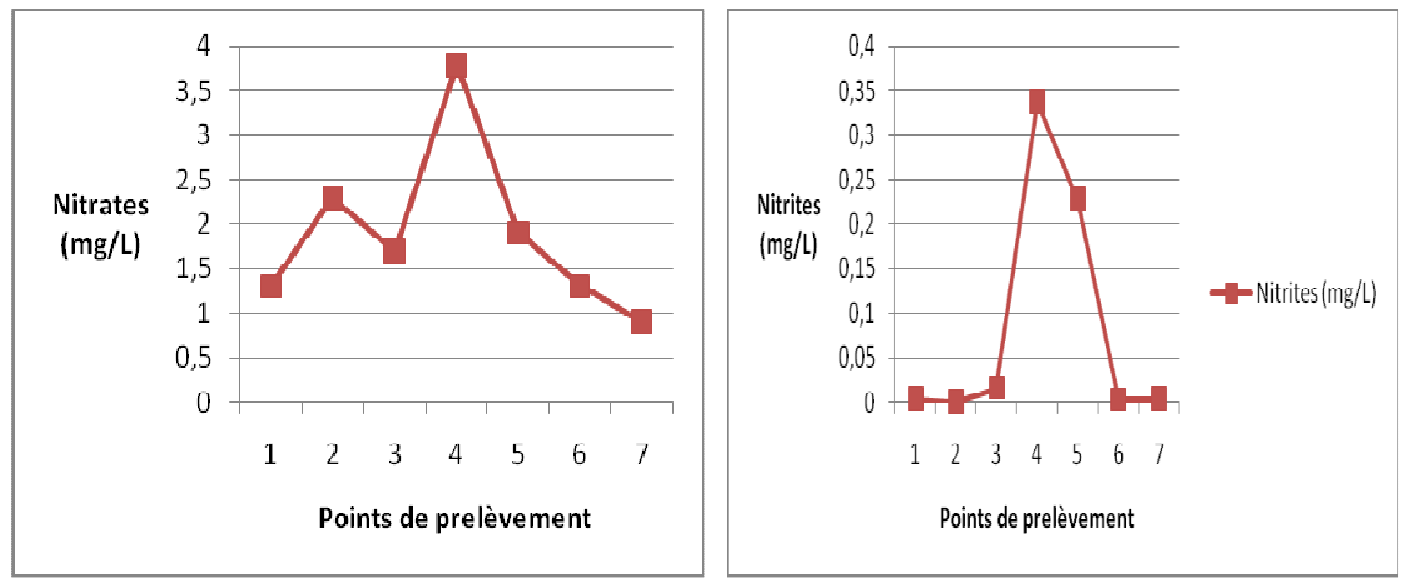

Figure 6: Valeurs des nitrates en fonction des points de prélèvement.

Figure 7: Valeurs des nitrites en fonction des points de prélèvement. 


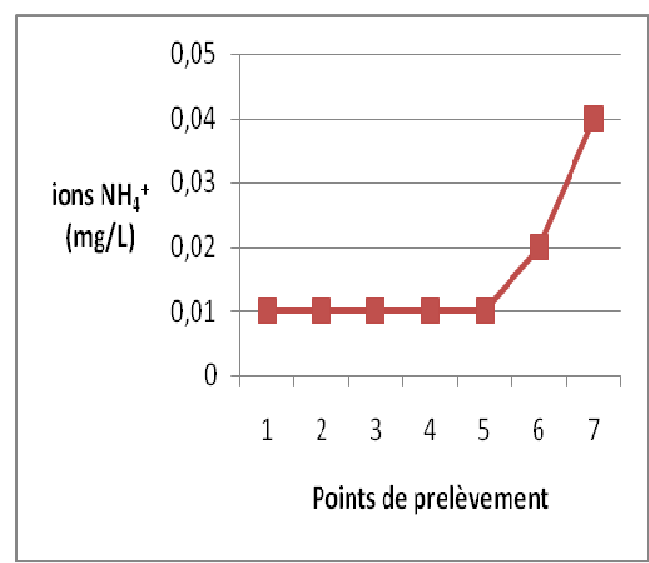

Figure 8: Valeurs des ions ammonium en fonction des points de prélèvement.

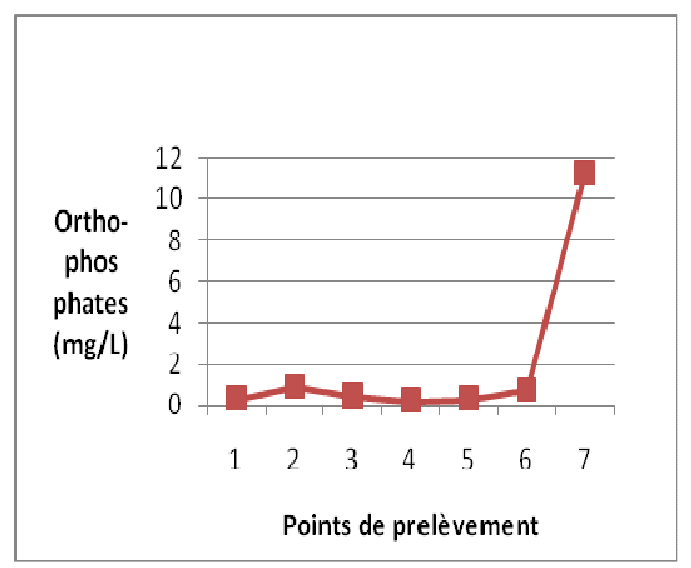

Figure 9: Valeurs des orthophosphates en fonction des points de prélèvement.
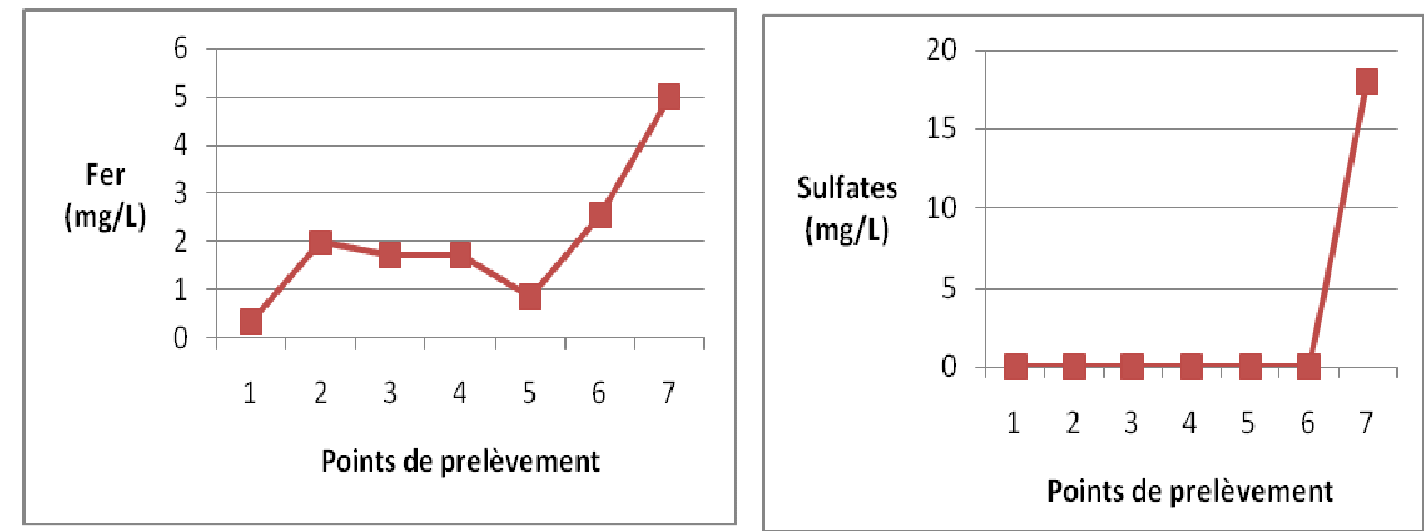

Figure 10: Valeurs du fer en fonction des points de prélèvement.
Figure 11: Valeurs des sulfates en fonction des points de prélèvement. 


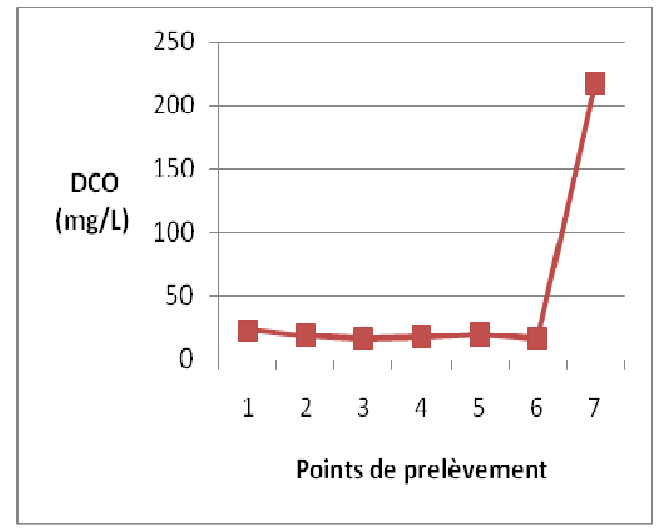

Figure 12 : Valeurs de la DCO en fonction des points de prélèvement.

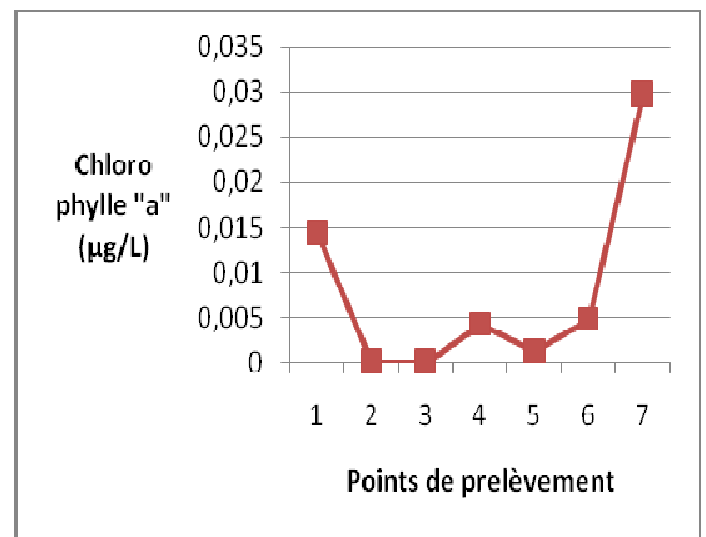

Figure 14 : Valeurs de la chlorophylle "a" en fonction des points de prélèvement.

\section{DISCUSSION}

\section{Interprétation des résultats}

Nous faisons ci-dessous les interprétations par type de paramètres, puis nous ferons un commentaire sur les paramètres hors normes (MEE décret 185, 2001 ; Belghyti, 2009), une analyse sur les différentes fonctions du marigot et enfin nous

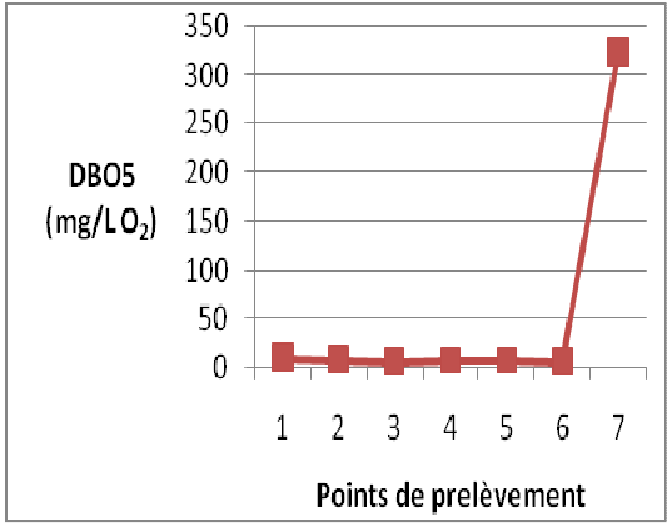

Figure 13 : Valeurs de la $\mathrm{DBO}_{5}$ en fonction des points de prélèvement.

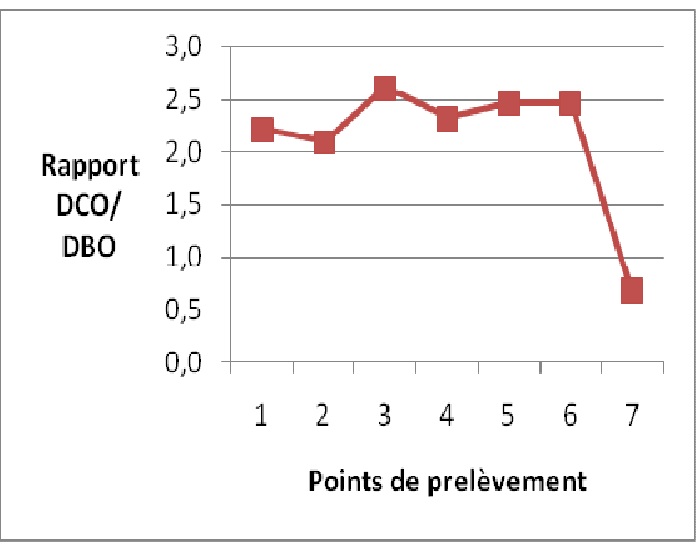

Figure 15: Valeurs du rapport DCO/DBO en fonction des points de prélèvement.

ferons une étude comparative de nos résultats avec ceux des années antérieures.

\section{Les paramètres physicochimiques}

Les Figures 1 à 3 indiquent les courbes représentant l'évolution de la température, du $\mathrm{pH}$ et de la conductivité avec les différents points de prélèvement. 
A partir des résultats présentés sur les Figures 1 à 3 ci-dessus, nous remarquons que le $\mathrm{pH}$ est proche de la neutralité. Les valeurs maximales se trouvent entre 7 et 7,2. Ces valeurs montrent l'absence d'une acidification du milieu. La conductivité est faible dans la partie amont du marigot et les valeurs varient entre 225 et $371 \mu \mathrm{S} / \mathrm{cm}$, mais elle a une valeur élevée pour l'échantillon P7 qui affiche une valeur de $1145 \mu \mathrm{S} / \mathrm{cm}$. Ce dernier est de fait loin de présenter une caractéristique des eaux de surface. Soulignons que c'est à ce point que les eaux traitées de la station de lagunage sont déversées dans le marigot, ce qui pourrait expliquer cette forte minéralisation.

Les Figures 4 à 15 ci-dessus indiquent les courbes représentant l'évolution des différents autres paramètres physicochimiques avec les points de prélèvement.

L'analyse portée sur ces résultats nous fait remarquer que : $15 \%$ des valeurs des résultats obtenus sont hors normes et concernent les paramètres suivants :

la conductivité sur l'échantillon P7 ;

les MES sur les échantillons P1 en

amont du pont Louveau, P4 à Dioulassoba et P7 ;

- $\quad$ les nitrites sur l'échantillon P4;

- $\quad$ le fer sur l'échantillon P5 à Warawara ;

- $\quad$ la DCO sur l'échantillon P7;

la $\mathrm{DBO}_{5}$ sur les échantillons $\mathrm{P} 1$ et $\mathrm{P} 2$

à l'est du Musée Communal, P4 et P5 ;

Environ $64 \%$ des paramètres physicochimiques présentent de très grands écarts de valeurs entre les prélèvements des points en aval P6 à l'ouest de la zone maraîchère de Dogona et P7 après l'exutoire de la Lagune et ceux en amont situés en milieu urbain (P1) et en amont du pont Louveau (P5) à Wara-wara.

En effectuant une comparaison entre les différents points de prélèvement, nous notons que les points $\mathrm{P} 4$ et $\mathrm{P} 7$ présentent le plus grand nombre de paramètres hors normes soit un total de quatre (4) tandis que les autres vont de un (1) sur les points P3, P5 et P6 à deux (2) pour les points P1 et P2. Le rapport DCO/DBO quant à lui est strictement inférieur à trois (3) avec des valeurs allant de 0,7 pour le point $\mathrm{P} 7$ après l'exutoire de la Lagune à 2,6 pour le point P3 à l'est du Jardin Zoologique. Cela caractérise une forte biodégradabilité de l'effluent.

\section{Les paramètres microbiologiques}

Les valeurs obtenues sur les paramètres microbiologiques, que nous avons indiqué dans le Tableau 2 ci-dessus, sont assez expressives sur la présence de matières fécales.

\section{Les paramètres organoleptiques}

Les résultats $\mathrm{du}$ Tableau 1 nous permettent de dire que : même si à certains endroits l'eau du marigot présente une coloration claire, (sites de prélèvements $\mathrm{P} 1$ en amont du pont Louveau, P3 à l'est du Jardin Zoologique et P6 à l'ouest de la zone maraîchère de Dogona), elle demeure trouble sur la majeure partie du marigot. Son odeur n'est pas assez perceptible sauf aux points P4 à Dioulassoba et P5 à Wara-wara où la perception est nette et devient forte au point P7 après l'exutoire de la Lagune.

On retrouve des déchets de grande importance à même le lit du marigot ainsi que de fréquentes traces de défécation le long du marigot. Les déchets plastiques y sont aussi présents. Dans sa partie avale, des débris de feuilles de légumes flottent à la surface de l'eau.

\section{Focus sur les paramètres hors normes}

Nous donnons ci-dessous les propriétés des paramètres dont les concentrations sont hors normes afin de montrer leurs conséquences sur les populations et l'environnement.

\section{La conductivité}

Elle estime la quantité de sels minéraux contenus dans l'eau. Son augmentation peut entraîner un problème de régulation chez certains organismes aquatiques sensibles, donne une limite à l'utilisation de l'eau comme source d'eau potable, une saveur impossible et occasionne des désordres digestifs. En l'absence de rejets 
industriels, les pollutions par minéralisation sont dues aux dépôts dans le lit du marigot. Des contrastes de conductivité (voir Figures 3 et 6 ci-dessus) sont observés et permettent de mettre en évidence des pollutions et surtout des zones de mélanges.

\section{Les Matières en Suspension (MES)}

Ce paramètre influe sur la turbidité de l'eau qui est inversement proportionnelle à sa transparence. La turbidité est la mesure de l'aspect plus ou moins trouble de l'eau. Elle est causée par diverses matières particulaires ou colloïdales composées de limon, d'argile, de composés organiques ou inorganiques ainsi que du plancton et d'autres microorganismes. Les sources de matières particulaires peuvent être d'origine naturelle (acides humiques, particules provenant de la dégradation des végétaux ou de l'érosion du sol) ou anthropique (rejets industriels, agricoles et urbains et surtout les offrandes faites aux silures).

\section{Les nitrites}

Ils proviennent essentiellement de la minéralisation de l'ammoniac en milieu oxydant (décomposition des déchets azotés) mais aussi des traitements de surface, des produits chimiques et des colorants. Ils disparaissent vite en milieu naturel. Leur présence constitue un indice de pollution. Le fer

Il provient du lessivage des dépôts d'ordures. Indispensable à la respiration et à la photosynthèse, il peut être un facteur limitant de la production par sa présence dans l'eau. En milieu alcalin, il peut compromettre la respiration des poissons et en milieu anaérobie il donne une couleur noire à l'eau. En valeur hors normes pour l'eau potable, il donne une coloration rouge à l'eau, un goût métallique et une odeur renvoyant à celle de poisson pourri.

\section{La DCO}

Elle traduit quantitativement et qualitativement la charge polluante organique.

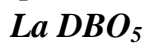

Elle est associée aux composés biodégradables ou bio-transformables. Ce focus met en évidence l'impact des dépôts d'ordures présents dans l'aire du marigot. Il fait pressentir le risque causé par la présence des déchets, des conséquences de leur décomposition et de leur contact permanent avec le cours d'eau. Les conséquences peuvent toucher aussi l'homme que la faune aquatique.

\section{Analyse à travers les fonctions du marigot \\ Le marigot Houët est d'une très grande utilité pour les populations riveraines. Malgré l'effort de couverture des besoins en service urbain de base dans le domaine de l'eau, l'hygiène et l'assainissement (MECV, 2010), les rôles socioéconomiques du Houët semblent irremplaçables pour la population autochtone. Pour notre analyse, nous avons classé ces fonctions en trois parties non exhaustives à savoir : eau domestique, eau de baignade et eau piscicole (MEE décret 185, 2001).}

\section{Eau du marigot Houët comme eau domestique}

Pour guider notre analyse, nous avons pris comme référence dans le décret cité en haut, son Article 7 sur les normes de qualité des eaux potabilisables, par degré de pollution et l'option A1 concernant le traitement physique simple et la désinfection. Pour certaines valeurs, nous nous sommes intéressés aux valeurs guides de l'OMS (OMS, 2004)

En comparant l'eau du marigot selon les usages faits aux points de prélèvements $\mathrm{P} 1$ en amont du pont Louveau, P2 à l'est du Musée Communal et P3 à l'est du Jardin Zoologique, à une eau potabilisable, nous notons que sur l'ensemble des paramètres évalués, ceux qui ne répondent pas aux normes sont: les MES $(+11 \mathrm{mg} / \mathrm{l})$ pour le point $\mathrm{P} 1$, le fer de $+0,23$ à $+1,88 \mathrm{mg} / \mathrm{L}$ sur les trois points sélectionnés et la $\mathrm{DBO}_{5}$ de $+3,5$ à $+7,4 \mathrm{mg} / \mathrm{L} \mathrm{O}_{2}$ sur les trois points sélectionnés. Les paramètres microbiologiques sont tous hors normes et cette remarque reste valable pour l'ensemble des analyses.

Les teneurs enregistrées en sulfates $\left(\mathrm{SO}_{4}{ }^{2-}\right)$, en nitrates $\left(\mathrm{NO}_{3}{ }^{-}\right)$et en nitrites $\left(\mathrm{NO}_{2}{ }^{-}\right)$ sont inférieures aux valeurs guides de l'OMS. 
- $\quad$ La croissance microbienne dans l'eau est particulièrement marquée à la surface des particules et à l'intérieur des flocs, naturellement présents dans l'eau ou formés lors de la coagulation. Ce phénomène résulte de l'adsorption d'éléments nutritifs aux surfaces, ce qui permet aux bactéries de croître plus efficacement. Des études antérieures ont mis en évidence un lien entre la turbidité et la présence de microorganismes (virus, bactéries et protozoaires) dans l'eau potable (Tarnagda et al., 2001; DRECV/HBs, 2007). La présence de bactéries pathogènes indique une souillure de l'eau par les matières fécales: présence d'excrétas humains et animaux. Une eau destinée à la consommation humaine ne doit pas contenir de germe indicateur d'une pollution fécale. Les populations de certains villages (Dioulasso-Bâ, Tounouma) ont aménagé des endroits dans le lit du marigot où ils puisent l'eau pour la boisson, la cuisine, la lessive, etc.... Au regard des résultats obtenus, le risque hydrique microbien est donc le plus évident pour ces populations. Pour l'essentiel, il se manifeste à court terme.

\section{Eau du marigot Houët comme eau de baignade}

Notre référence dans le décret cité est l'Article 8 sur les normes de qualité des eaux de baignade (MEE décret 185, 2001). Les sites de prélèvements concernés sont $\mathrm{P} 4$ à Dioulassoba et P6 à l'ouest de la zone maraîchère de Dogona.

Les paramètres hors normes sont les MES du point $\mathrm{P} 4$ avec $+03 \mathrm{mg} / \mathrm{L}$, le fer avec $+1,61 \mathrm{mg} / \mathrm{L}$ et $+2,45 \mathrm{mg} / \mathrm{L}$ et $1 \mathrm{a} \mathrm{DBO}_{5}$ avec $+3,7$ et $+4,8 \mathrm{mg} / \mathrm{L} \mathrm{O}_{2}$.

Le taux élevé de fer ainsi que la présence de germes bactériologiques dégradent la qualité des eaux et peuvent conduire à des affections de santé, le plus souvent bénignes, par contact cutané ou compte tenu de la possibilité d'ingérer ou d'inhaler de l'eau.

L'observation ci-dessus sur les paramètres microbiologiques reste valable. En l'état, la pollution microbiologique des eaux du marigot Houët dans la fonction d'eau de baignade est essentiellement d'origine fécale. En effet, les eaux usées provenant des habitations, les déjections des animaux et les effluents d'élevage rejetés dans le milieu polluent les sites de baignades et peuvent être la cause d'une mauvaise qualité de l'eau.

Eau du marigot Houët comme eau piscicole Notre référence dans le décret cité est l'Article 9 sur les normes pour la protection de la qualité des eaux piscicoles, option eaux cyprinicoles et valeurs imposées (MEE décret 185, 2001). Les sites de prélèvements concernés sont P1 en amont du pont Louveau, P2 à l'est du Musée Communal et P4 à Dioulassoba. Ces sites abritent des mares aux silures sacrées et sont très usités comme sites d'offrandes.

Seuls deux (2) paramètres sont critiques ici à savoir les nitrites au point $\mathrm{P} 4$ avec une valeur de $+0,3 \mathrm{mg} / \mathrm{l}$ supérieure à la norme et la $\mathrm{DBO}_{5}$ qui affiche respectivement $+4,4 \mathrm{mg} / \mathrm{L} \mathrm{O}_{2}$ au point $\mathrm{P} 1,+3,2 \mathrm{mg} / \mathrm{L} \mathrm{O}_{2}$ au point $\mathrm{P} 2$ et $+1,8 \mathrm{mg} / \mathrm{L} \mathrm{O}_{2}$ au point $\mathrm{P} 4$.

La protection des eaux de surface du marigot Houët par les autorités en collaboration avec la population à travers un comité de suivi mis en place par Arrêté Municipal (AMCB 049, 2006) a, entre autres, pour objectif de maintenir la qualité physicochimique de celles-ci pour permettre la vie des poissons sacrés. La survie de ceux-ci dépend d'une série de paramètres comme la présence d'oxygène ou encore l'absence de toxiques (nitrites, métaux). Notre étude n'a pas concerné suffisamment de valeurs pour mieux apprécier cette fonction à ne point négliger vu le rôle que ces dits poissons sacrés jouent dans le dispositif de maintien de l'écosystème du marigot. En effet, à travers leurs va-et-vient entre la partie superficielle et souterraine du marigot, ils contribuent à déboucher les interstices et à maintenir l'alimentation du cours d'eau par des sources émergentes. Ces sources contribuent à augmenter la capacité de dilution de l'effluent. Au-delà, ces poissons contribuent à l'épuration naturelle des eaux du marigot et le rapport $\mathrm{DCO} \mathrm{DBO}_{5}$ étant 
équivalent à 2 sur l'ensemble de ces trois points, la qualité biodégradable de l'effluent leur est favorable.

\section{Etude comparative de nos résultats avec ceux des années antérieures}

En 2001 et 2007, des études avaient été effectuées pour déterminer l'état de pollution du marigot Houët (Tarnagda et al., 2001 DRECV/HBs, 2007). Le Tableau 3 contient les résultats de ces études antérieures sur les valeurs de la température, du $\mathrm{pH}$, de la conductivité et de la turbidité ainsi que les nôtres obtenues en 2012. Ces résultats montrent que :

- les valeurs de la température que nous avons obtenu en 2012 sont inférieures aux valeurs de 2001 et de 2007, mais celles du pH n'ont pas varié car elles sont les mêmes aussi bien en 2012, en 2001 qu'en 2007 ;

- les valeurs de la conductivité obtenues en 2012 sont très élevées surtout en zone avale par rapport à celles obtenues en 2001 et 2007. Il en est de même pour la turbidité.

On peut donc dire qu'il y a eu une augmentation de la pollution domestique, surtout en zone avale avec le temps.

\section{Conclusion}

Le but de notre étude était de faire un état des lieux sur le plan de la pollution du marigot afin de contribuer à la protection de son écosystème et à l'amélioration de la santé et du cadre de vie des populations riveraines. L'étude a révélé que l'eau du marigot Houët est restée très polluée par les effluents d'origines domestique et agricole, malgré la mise en fonctionnement d'égouts dans la zone amont et d'une station d'épuration par lagunage à Dagona en 2008 (éléments nouveaux depuis 2007) qui a permis une diminution de la pollution industrielle (rapport DCO/DBO strictement inférieur à 3 caractérisant une forte biodégradabilité de l'effluent). Cette eau ne convient ni à une utilisation domestique, ni au développement d'une baignade (les MES, le fer et la $\mathrm{DBO}_{5}$ ainsi que les paramètres microbiologiques sont tous hors normes), ni au milieu de vie des poissons (les nitrites et la $\mathrm{DBO}_{5}$ sont hors normes). Aussi, nos recommandations sont les suivantes :

- Vu l'importance sociale des silures, qualifiés «d'êtres patrimoniaux et irremplaçables », les autorités communales devraient prendre des mesures de conservation à leur égard :

- $\mathrm{Vu}$ le danger que constitue la pollution de la rivière, d'une part pour la population, d'autre part pour les silures sacrés et autrement pour le Kou et le Mouhoun, les autorités communales devraient assainir cette rivière et y mener des actions de sauvegarde et de valorisation plus élaborées et plus dynamiques qui lui garantiraient un meilleur devenir ;

- à court terme, la zone amont devrait être mise sous contrôle d'hygiène et la zone avale sous surveillance contre le transfert de pollution.

\section{REFERENCES}

AEM. 2012. Rapport d'Etat des lieux de l'espace de compétence de l'Agence de l'eau du Mouhoun (AEM). Version Intermédiaire, Dédougou, Burkina Faso.

AMCB 049. 2006. Arrêté Municipal N²006 049/CB/M/CAB de la Commune de Bobo-Dioulasso portant création, attribution, composition et fonctionnement du Comité de Suivi du marigot Houët à Bobo-Dioulasso.

Belghyti D, El Guamri Y, Ztit G, Ouahidi LM, Joti MB, Harchrass A, Amghar H, Bouchouata O, Kaharrim K, Bounouira H. 2009. Caractérisation physicochimique des eaux usées d'abattoir en vue de la mise en oeuvre d'un traitement adéquat : cas de Kénitra au Maroc. Afrique Science, 5(2): 199 - 216.

Banhoro C. 2007. La pollution du marigot Houët et ses conséquences potentielles sur l'environnement. Mémoire de fin de cycle présenté en vue de l'obtention du diplôme de contrôleur des eaux et eorêts, Ecole Nationale des Eaux et Forêts, Bobo-Dioulasso, Burkina Faso. 
DGACV/MECV. 2005. Rapport du constat et d'enquête sur la pollution du marigot Houët le 21 juin 2005. Direction générale de l'Assainissement et du Cadre de Vie / Ministère de l'Environnement et du Cadre de Vie (DGACV/MECV), Burkina Faso.

DRECV/HBs. 2007. Constat et investigation sur la mortalité des silures sacrés du marigot Houët les 11 et 17 août 2007 à Bobo-Dioulasso. Direction Générale de l'Environnement et du Cadre de Vie / Région des Hauts Bassins (DRECV/HBs).

Mama D, Matchi B, Aina M, Maman -Sanni I, Moudachirou M, Matejka G. 2010. Impact d'une décharge des déchets solides ménagers sur la ressource en eau : étude de cas. Bulletin d'Informations de la SOACHIM, 7: 1 -14.

MECV. 2010. Stratégie communale de gestion des services urbains de base dans les domaines de l'eau, de l'hygiène et de l'assainissement du Ministère de
l'Environnement et du Cadre de Vie (MECV), Burkina Faso.

MEE décret 185. 2001. Décret $n^{\circ}$ 2001-185 du 7 mai 2001 portant fixation des normes de rejets de polluant dans l'air, l'eau et le sol.

OMS. 2004. Normes de l'OMS de 2004.

Tarnagda Z, N'Diaye S, Ido G, Berte D, Bassolet K, Cogels X, Rossillon F. 2001. Communication aux JSS BoboDioulasso : les eaux usées domestiques et industrielles de la ville de BoboDioulasso: une menace sanitaire et écologique?

Wellens J, Sawadogo I, Diallo M, Dakouré D, Compaoré NF, Traoré F, Tychon B. 2007. Recensement exhaustif des activités hydro-agricoles du Bassin du Kou. Projet GEeau, Burkina Faso. (www.ge-eau.org). 